\title{
Retraction
}

\section{Retracted: Design of Exercise Nursing Program for Pelvic Floor Muscle Function Recovery at 42 Days Postpartum}

\author{
Computational and Mathematical Methods in Medicine \\ Received 19 November 2022; Accepted 19 November 2022; Published 29 December 2022 \\ Copyright ( $) 2022$ Computational and Mathematical Methods in Medicine. This is an open access article distributed under the \\ Creative Commons Attribution License, which permits unrestricted use, distribution, and reproduction in any medium, \\ provided the original work is properly cited.
}

Computational and Mathematical Methods in Medicine has retracted the article titled "Design of Exercise Nursing Program for Pelvic Floor Muscle Function Recovery at 42 Days Postpartum" [1] due to concerns that the peer review process has been compromised.

Following an investigation conducted by the Hindawi Research Integrity team [2], significant concerns were identified with the peer reviewers assigned to this article; the investigation has concluded that the peer review process was compromised. We therefore can no longer trust the peer review process and the article is being retracted with the agreement of the Chief Editor.

The authors do not agree to the retraction.

\section{References}

[1] S. Zhang, L. Yuan, L. Zhou, X. Lei, and L. Zhu, "Design of Exercise Nursing Program for Pelvic Floor Muscle Function Recovery at 42 Days Postpartum," Computational and Mathematical Methods in Medicine, vol. 2021, Article ID 1714610, 10 pages, 2021.

[2] L. Ferguson, "Advancing Research Integrity Collaboratively and with Vigour," 2022, https://www.hindawi.com/post/advancingresearch-integrity-collaboratively-and-vigour/. 


\title{
Design of Exercise Nursing Program for Pelvic Floor Muscle Function Recovery at 42 Days Postpartum
}

\author{
Shuixian Zhang, Ling Yuan (D), Lin Zhou, Xia Lei, and Libo Zhu \\ Department of Obstetrics, Affiliated Hangzhou First People's Hospital, Zhejiang University School of Medicine, Zhejiang, \\ Hangzhou 310006, China \\ Correspondence should be addressed to Ling Yuan; 2017a600@zcmu.edu.cn
}

Received 23 September 2021; Revised 6 October 2021; Accepted 1 November 2021; Published 10 November 2021

Academic Editor: Osamah Ibrahim Khalaf

Copyright (C) 2021 Shuixian Zhang et al. This is an open access article distributed under the Creative Commons Attribution License, which permits unrestricted use, distribution, and reproduction in any medium, provided the original work is properly cited.

\begin{abstract}
In order to explore the effect of exercise nursing intervention for pelvic floor muscle function recovery at 42 days postpartum, this paper conducts experimental research through controlled experiments, combines statistical methods to carry out digital processing, and sets a single variable of nursing intervention to provide a basis for experimental control, and statistical test results are used for effect evaluation. It has been discovered via experimental study that pelvic floor muscle function recovery exercise training for normal delivery women may enhance the mother's compliance with the pelvic floor muscle function exercise. Moreover, it can reduce the occurrence of urinary incontinence and pelvic organ prolapse, improve the postpartum pelvic floor function of postpartum women, improve the quality of life, and have a significant recovery effect. In addition, it is a simple, noninvasive, and highly safe continuation nursing measure, which can effectively improve the quality of obstetric care and has strong clinical use value.
\end{abstract}

\section{Introduction}

Female pelvic floor dysfunction is also known as pelvic floor defect or pelvic floor support tissue laxity, and the incidence of this disease in middle-aged and elderly women is about $20 \%-40 \%$. Various causes such as pregnancy, childbirth, pelvic floor surgery, drugs, and estrogen deficiency lead to weak pelvic floor support, which in turn leads to the displacement of pelvic organs and causes abnormal positions and functions of other pelvic organs. Among them, the primary reason is the structural damage of the pelvic floor caused by pregnancy and childbirth [1].

The compliance of pelvic floor muscle training is affected by many factors, mainly affected by the patient's own factors, physical conditions, and medical and socioeconomic conditions, among which patient factors are the main factors affecting the compliance of pelvic floor muscle training. In recent years, a large number of foreign studies have focused on the compliance of pelvic floor muscle training in patients with urinary incontinence. Lack of time, forgetting to train, cognitive level, PFMT attitude, self-efficacy, illness level, age, education, and ambivalence about the usefulness of PFMT are the major variables that influence compliance with pelvic floor muscle training [2]. The incidence of postpartum urinary incontinence is very high. Many women during pregnancy and postpartum believe that urinary incontinence is a natural phenomenon of pregnancy and childbirth; so, they will not actively take preventive measures, but the early changes caused by pregnancy and childbirth are the biochemical and electrophysiological changes of the pelvic floor supporting tissues. If there is no prevention and treatment, the injury will develop further, and symptomatic pelvic floor dysfunction diseases will appear. Therefore, the prevention and treatment of urinary incontinence should start during pregnancy and after delivery. If mothers do not actively prevent and treat urinary incontinence after delivery, the incidence of long-term urinary incontinence will increase. A retrospective study of 3599 elderly women found that the incidence of long-term urinary incontinence in women with postpartum urinary incontinence was $40 \%$. In addition, postpartum women are in the transition period, psychological and physiological are in the adaptation period, the environment, mood, and disease conditions of patients with urinary 
incontinence in other periods are different, and the influencing factors may also be different from those of women with urinary incontinence in other periods. At this stage, the research on the compliance of pelvic floor muscle training in women with urinary incontinence has become a hot research topic, but there are relatively few articles focusing on the compliance of pelvic floor muscle training in women with postpartum urinary incontinence and its influencing factors. Therefore, it is very necessary to actively explore the pelvic floor muscle training compliance of women with postpartum urinary incontinence and its influencing factors to provide theoretical guidance for future pelvic floor muscle training intervention [3].

Physiological changes during pregnancy and childbirth cause increased abdominal pressure and decreased collagen in the supporting structure of the pelvic floor, which reduces the muscle strength of the pelvic floor and relaxes the supporting tissues of the pelvic floor. This is a prone stage of pelvic floor dysfunction diseases. The fetal head exerts continuous mechanical compression and expansion on the pelvic floor muscles and nerves as the fetal presentation decreases during childbirth, causing these muscles and nerves to be stretched and damaged, leading to defects in the function of the muscle fibres they innervate, and the second stage of labor is prolonged. The mechanical compression and expansion of the fetal head on the pelvic floor muscles and nerves will last longer during operations to assist delivery and delivery of giant fetuses, and the damage will be more intense, beyond the range that physiological changes can adapt, causing irreversible pelvic floor tissue structure damage. Another study on the movement and rotation angle of the bladder neck of primiparas has shown that childbirth increases the movement of the bladder neck, and the incidence of postpartum urinary incontinence increases significantly in those who have an increase in the movement of the bladder neck before delivery.

Vaginal delivery is a special physiological process for women. During the process of delivery, the contraction tension of the pelvic floor muscles of the parturient is significantly reduced, and some damage is caused. Postpartum pelvic floor dysfunction is often accompanied by varying degrees of pelvic floor dysfunction, aggravating the parturient's physical and emotional discomfort and impairing her postpartum recovery. The importance of early scientific and successful treatments following vaginal birth in supporting the healing of pelvic floor muscles cannot be overstated. Postpartum exercises formulate a targeted functional training program based on the physical condition of the parturient and guide the parturient to perform hip exercises and twisting exercises. Continuous and standardized pelvic floor muscle training can accelerate the recovery of muscle fibers. On this basis, pelvic floor rehabilitation care is taken and given to the patient. Electrical stimulation and biofeedback intervention can produce electrical stimulation to the pelvic floor muscles, aiming to better promote the recovery of maternal pelvic floor function.

\section{Related Work}

Midwives should continue to urge women to increase the frequency and consistency of pelvic floor exercises following delivery, according to the research [4]. From the basic Kegel exercise to pelvic floor rehabilitation, foreign nations have developed a set of scientific, effective, safe, and mature therapeutic techniques for female pelvic floor rehabilitation. The parturient's pelvic floor muscle function exercise was included in the nursing routine in a literature review [5]. Increased nursing staff attention increased the parturient's exercise awareness and behavior, resulting in better executive ability of the puerperium women to adhere to the pelvic floor function exercise. The literature [6] made the mothers aware of the causes and prevention methods of postpartum urinary incontinence, emphasized the importance of early postpartum pelvic floor muscle training, and enabled the mothers to actively carry out pelvic floor muscle training through various forms of health education activities. The literature [7] placed the health education on pelvic floor function rehabilitation across the whole pregnancy-childbirth-puerperium by enhancing health education and popularising information of female pelvic floor dysfunction disorders. Pelvic floor muscle rehabilitation exercises done 42 days after birth are beneficial in avoiding pelvic floor dysfunction problems, according to study [8]. In clinical practice, however, many parturients do not have a clear knowledge of the risks of pelvic floor dysfunction illnesses and Kegel exercise, resulting in Kegel exercise not being extensively used. Furthermore, even if they have pelvic floor dysfunction, some parturients are shy, making it difficult for them to speak out (such as postpartum urine incontinence). According to the research [9], external variables such as monotonous Kegel movement, set exercise mode, and lengthy exercise duration make it difficult to continue exercising. According to the literature [10], only $38 \%$ of parturients can continue with Kegel exercise and have low compliance. In 1948, an American physician named Arnold Kegel created Kegel exercise as an active pelvic floor rehabilitation method. Pelvic floor muscular strength is restored by contracting and relaxing the pelvic floor muscles on a regular basis. Kegel exercise has been shown to be a simple, straightforward, painless, and effective technique after years of clinical experience [11]. The majority of women, particularly postpartum women, are unaware of pelvic floor dysfunction illnesses. Furthermore, some medical personnel at community hospitals paid little attention to the illness and failed to establish a comprehensive pelvic floor exercise instruction program for perinatal women [12].

At present, a large number of studies have focused on the role of pelvic floor muscle training in pelvic floor disorders, especially the role of pelvic floor muscle training in the prevention and treatment of urinary incontinence. However, there are few studies on whether pelvic floor muscle training can prevent and treat other pelvic floor disorders, such as fecal incontinence. In addition, there are disagreements on whether prenatal pelvic floor muscle training can prevent the occurrence of postpartum urinary incontinence. Literature [13] conducted a randomized controlled trial on 855 pregnant women and conducted 12 weeks of pelvic floor muscle training in the intervention group. It was found that pelvic floor muscle training before delivery did not significantly reduce the incidence of postpartum urinary incontinence. However, prenatal pelvic floor muscle training can improve the outcome of childbirth, especially shorten the time of labor, and reduce the mechanical compression of the pelvic floor muscles during 
childbirth, thereby reducing the occurrence of urinary incontinence. The American College of Obstetrics and Gynecology (ACOG) committee believes that in the absence of contraindications, pregnant women should be encouraged to participate in regular PFMTn041. PFMT during pregnancy is beneficial to both the body and mind of pregnant women. It can promote blood circulation in the pelvic floor of pregnant women, strengthen the muscle tension of the pelvic floor, reduce the compression of the uterus of pregnancy on the veins of the lower extremities, reduce fat deposits in the pelvis, and adjust the state of mind. Moreover, it is conducive to natural delivery, reduces the occurrence of dystocia such as cesarean section and vaginal delivery, and also helps prevent stress urinary incontinence, uterine prolapse, postpartum sexual dysfunction, and other diseases [14].PFMT may also increase pelvic floor muscle strength, toughness, and suppleness during pregnancy. The extension and contraction of the pelvic floor muscles may aid the fetal presenting portion spin inside the pelvic cavity and enable the fetal head to expand and deliver when the labor process reaches the active phase [15]. According to the literature [16], when pregnant women receive PFMT between 20 and 36 weeks of pregnancy, the active phase of labor may be expedited to reach the second stage of labor during delivery, reducing the total birth time. Continuous prenatal pelvic floor muscle training may substantially reduce the time spent in the second stage of labor, according to the research [17]. At the same time, the research suggests that PFMT behavior during pregnancy may aid in the rehabilitation of postpartum pelvic floor muscle strength. Continuous pelvic floor muscle training before to delivery may aid in the rehabilitation of pelvic floor function after childbirth, decreasing urine incontinence. Various types of pelvic floor muscle training treatments have varied impacts on the incidence of urine incontinence during pregnancy or after delivery. The pelvic floor muscle training strategy in the literature [18] began 48 hours after delivery and lasted eight weeks. According to the results, strengthening the pelvic floor muscles may significantly reduce the incidence of postpartum urinary incontinence. The incidence of urinary incontinence was significantly reduced in the intervention group at 6 and 9 months postpartum, according to the results of a research [19] that intervened in pelvic floor muscle training for parturients $10-16$ weeks postpartum. The literature [20] utilized a single-blind randomized controlled trial to split the participants into two groups: pelvic floor muscle training and general fitness, with the pelvic floor muscle training intervention taking place between 24 weeks of pregnancy. There was no significant change in the incidence of urine incontinence during pregnancy, according to the findings.

\section{Research Method}

This article selects the primiparous women who were in fullterm head position in a hospital from January 2019 to June 2021 and divides them into a control group and an observation group according to a random number table. The maternal age of the control group is 21-34 years old, and the average age is $(24 \pm 4.1)$ years, the newborn weight is 2522 $3992 \mathrm{~g}$, and the average weight is $3438 \pm 324 \mathrm{~g}$. The maternal age in the observation group is 22-35 years old, and the average age is $27 \pm 4.2$ years. The newborn's weight is 2 $496-4175 \mathrm{~g}$, and the average is $3418 \pm 333 \mathrm{~g}$. There is no significant difference in maternal age and newborn weight between the two groups $(P>0.05)$, and they are comparable: full-term singleton pregnancy, no problems during pregnancy or puerperium, no organ illnesses (heart, liver, or kidney), no history of pelvic surgery, and unaware mental disorder. This study is approved by the parturient and signed an informed consent form.

During the hospitalization period, both the control and observation groups provided postpartum care to the parturient according to the routine postpartum nursing model, which included psychological care, vaginal secretion observation, perineal care, diet, and postpartum rehabilitation knowledge education. Nurses need to guide the pelvic floor muscle function rehabilitation training of the parturient every day. When discharged from the hospital, it is necessary to issue a pelvic floor muscle rehabilitation training nursing manual, instruct the mother to continue to adhere to the pelvic floor muscle rehabilitation training after discharge from the hospital, and follow up if there is any discomfort. On the basis of the above measures, the observation group carries out continuous Kegel exercise training nursing guidance for discharged women, which includes the following: (1) it is necessary to set up a 7person continuation nursing team consisting of 2 nurses in charge, 3 nurses, and 2 nurses. The team members are all undergraduates and have good communication and coordination skills. (2) The head nurse divides the work of the team members and organizes the members to formulate the continuation care plan, the continuation of the Kegel exercise program, and the evaluation criteria for pelvic floor function rehabilitation. (3) The team members need to be trained in postpartum rehabilitation knowledge, knowledge of preventing complications, and continuation of Kegel exercise-related knowledge. Kegel exercise training includes rhythmic vaginal contraction and relaxation exercises, hip contraction and levator anal exercises, and abdominal contraction and contraction of the inner thigh muscles. Each exercise is 3-4 times a day, and each sport is trained 10-20 times each time. The amount of exercise is adjusted according to the individual. (4) The patient's name, age, residence, phone number, QQ, or WeChat, as well as pelvic floor muscle rehabilitation exercise training, urine incontinence, pelvic organ prolapse, and other occurrences, must all be included in the information files of discharged moms. (5) It is necessary to continue the nursing method and frequency. It is necessary to establish a contact channel with the parturient through telephone, QQ, or WeChat. One week after discharge, the first telephone followup needs to be completed and once a week in the first month after discharge. After discharge from the hospital, the followup will be carried out once every two weeks and once a month for 4-6 months. The frequency of follow-up can be adjusted according to the needs of the patient.(6) Six months after discharge, the incidence of pelvic organ prolapse, the incidence of urinary incontinence, the compliance of pelvic floor exercises, and the rehabilitation effect of pelvic floor muscle tension of the two groups of parturient are evaluated.

On this basis, the control group also adopts the following nursing methods: 
TABLE 1: Comparison of pelvic floor comprehensive muscle strength.

\begin{tabular}{|c|c|c|c|c|c|c|c|c|}
\hline Number & Control group & Test group & Number & Control group & Test group & Number & Control group & Test group \\
\hline 1 & 40.91 & 72.10 & 21 & 55.45 & 68.75 & 41 & 41.33 & 58.06 \\
\hline 2 & 60.83 & 56.01 & 22 & 63.66 & 31.23 & 42 & 60.26 & 47.69 \\
\hline 4 & 32.96 & 35.12 & 24 & 48.80 & 35.03 & 44 & 32.74 & 35.25 \\
\hline 5 & 38.18 & 34.20 & 25 & 46.75 & 52.48 & 45 & 60.32 & 58.10 \\
\hline 6 & 28.02 & 61.39 & 26 & 71.95 & 33.47 & 46 & 41.96 & 49.93 \\
\hline 8 & 49.22 & 62.13 & 28 & 37.89 & 43.85 & 48 & 29.12 & 41.17 \\
\hline 9 & 52.56 & 79.90 & 29 & 73.95 & 44.42 & 49 & 34.75 & 63.48 \\
\hline 10 & 25.13 & 36.15 & 30 & 68.09 & 71.95 & 50 & 28.94 & 81.71 \\
\hline 11 & 29.83 & 36.87 & 31 & 46.26 & 31.68 & 51 & 27.05 & 55.46 \\
\hline 12 & 62.27 & 63.19 & 32 & 70.78 & 34.18 & 52 & 45.69 & 58.15 \\
\hline 16 & 37.78 & 46.90 & 36 & 62.73 & 71.89 & 56 & 56.71 & 83.20 \\
\hline 17 & 69.15 & 51.83 & 37 & 73.50 & 67.86 & 57 & 68.59 & 78.42 \\
\hline 18 & 73.53 & 66.52 & 38 & 59.09 & 43.07 & 58 & 54.56 & 77.86 \\
\hline 19 & 66.35 & 50.74 & 39 & 30.44 & 38.33 & 59 & 31.04 & 44.39 \\
\hline 20 & 31.03 & 46.66 & 40 & 58.57 & 36.21 & 60 & 66.66 & 77.55 \\
\hline
\end{tabular}

TABLE 2: Comparison of the incidence of urinary incontinence.

\begin{tabular}{|c|c|c|c|c|c|c|c|c|}
\hline Number & Control group & Test group & Number & Control group & Test group & Number & Control group & Test group \\
\hline 1 & 0.089 & 0.064 & 21 & 0.082 & 0.066 & 41 & 0.097 & 0.055 \\
\hline 2 & 0.098 & 0.072 & 22 & 0.091 & 0.078 & 42 & 0.099 & 0.073 \\
\hline 3 & 0.086 & 0.077 & 23 & 0.099 & 0.069 & 43 & 0.100 & 0.079 \\
\hline 4 & 0.081 & 0.056 & 24 & 0.091 & 0.074 & 44 & 0.093 & 0.074 \\
\hline 5 & 0.088 & 0.076 & 25 & 0.090 & 0.074 & 45 & 0.088 & 0.067 \\
\hline 6 & 0.097 & 0.060 & 26 & 0.091 & 0.067 & 46 & 0.092 & 0.051 \\
\hline 7 & 0.081 & 0.059 & 27 & 0.089 & 0.071 & 47 & 0.084 & 0.051 \\
\hline 8 & 0.093 & 0.078 & 28 & 0.085 & 0.070 & 48 & 0.087 & 0.067 \\
\hline 9 & 0.084 & 0.067 & 29 & 0.096 & 0.059 & 49 & 0.095 & 0.073 \\
\hline 10 & 0.083 & 0.079 & 30 & 0.082 & 0.054 & 50 & 0.087 & 0.075 \\
\hline 11 & 0.086 & 0.066 & 31 & 0.082 & 0.057 & 51 & 0.099 & 0.073 \\
\hline 12 & 0.093 & 0.059 & 32 & 0.086 & 0.069 & 52 & 0.092 & 0.059 \\
\hline 13 & 0.094 & 0.052 & 33 & 0.084 & 0.053 & 53 & 0.089 & 0.075 \\
\hline 14 & 0.094 & 0.079 & 34 & 0.091 & 0.077 & 54 & 0.098 & 0.066 \\
\hline 15 & 0.090 & 0.072 & 35 & 0.085 & 0.071 & 55 & 0.094 & 0.069 \\
\hline 16 & 0.083 & 0.073 & 36 & 0.086 & 0.052 & 56 & 0.086 & 0.064 \\
\hline 17 & 0.085 & 0.063 & 37 & 0.095 & 0.067 & 57 & 0.094 & 0.074 \\
\hline 18 & 0.088 & 0.075 & 38 & 0.093 & 0.076 & 58 & 0.086 & 0.050 \\
\hline 19 & 0.098 & 0.058 & 39 & 0.085 & 0.053 & 59 & 0.089 & 0.070 \\
\hline 20 & 0.092 & 0.074 & 40 & 0.097 & 0.072 & 60 & 0.089 & 0.076 \\
\hline
\end{tabular}

It is essential to provide postpartum regular care, such as the pelvic floor muscle rehabilitation knowledge handbook, and to provide perineal care and nutrition advice after delivery. Simultaneously, start postpartum pelvic floor muscle recovery exercises on the second day after birth, add 1 segment every 1-2 days, perform 8-16 times each session, and maintain pelvic floor muscle recovery exercises after discharge until 6 weeks postpartum. For second-born mothers 
TABLE 3: Comparison of the incidence of pelvic organ prolapse.

\begin{tabular}{|c|c|c|c|c|c|c|c|c|}
\hline Number & Control group & Test group & Number & Control group & Test group & Number & Control group & Test group \\
\hline 1 & 0.066 & 0.030 & 21 & 0.072 & 0.038 & 41 & 0.060 & 0.055 \\
\hline 2 & 0.056 & 0.021 & 22 & 0.051 & 0.021 & 42 & 0.055 & 0.053 \\
\hline 4 & 0.067 & 0.042 & 24 & 0.059 & 0.038 & 44 & 0.067 & 0.027 \\
\hline 5 & 0.068 & 0.025 & 25 & 0.061 & 0.054 & 45 & 0.057 & 0.025 \\
\hline 6 & 0.064 & 0.028 & 26 & 0.051 & 0.026 & 46 & 0.068 & 0.026 \\
\hline 8 & 0.069 & 0.020 & 28 & 0.065 & 0.036 & 48 & 0.072 & 0.031 \\
\hline 9 & 0.052 & 0.036 & 29 & 0.078 & 0.046 & 49 & 0.052 & 0.035 \\
\hline 10 & 0.057 & 0.039 & 30 & 0.079 & 0.046 & 50 & 0.058 & 0.045 \\
\hline 11 & 0.075 & 0.027 & 31 & 0.063 & 0.041 & 51 & 0.063 & 0.048 \\
\hline 12 & 0.080 & 0.023 & 32 & 0.066 & 0.027 & 52 & 0.057 & 0.041 \\
\hline 16 & 0.077 & 0.025 & 36 & 0.076 & 0.028 & 56 & 0.065 & 0.034 \\
\hline 17 & 0.068 & 0.025 & 37 & 0.067 & 0.058 & 57 & 0.063 & 0.023 \\
\hline 18 & 0.074 & 0.046 & 38 & 0.068 & 0.051 & 58 & 0.071 & 0.054 \\
\hline 19 & 0.053 & 0.048 & 39 & 0.075 & 0.049 & 59 & 0.056 & 0.040 \\
\hline 20 & 0.064 & 0.047 & 40 & 0.068 & 0.050 & 60 & 0.074 & 0.048 \\
\hline
\end{tabular}

TABLE 4: Comparison of FSFI comprehensive score.

\begin{tabular}{|c|c|c|c|c|c|c|c|c|}
\hline Number & Control group & Test group & Number & Control group & Test group & Number & Control group & Test group \\
\hline 1 & 60.94 & 79.89 & 21 & 76.80 & 72.86 & 41 & 61.75 & 81.69 \\
\hline 2 & 67.00 & 83.26 & 22 & 73.87 & 78.60 & 42 & 79.68 & 66.70 \\
\hline 3 & 63.63 & 87.11 & 23 & 78.53 & 78.15 & 43 & 78.40 & 88.05 \\
\hline 4 & 66.18 & 84.80 & 24 & 72.43 & 84.55 & 44 & 60.23 & 87.49 \\
\hline 5 & 63.69 & 75.82 & 25 & 64.61 & 85.13 & 45 & 60.05 & 70.35 \\
\hline 6 & 81.65 & 83.11 & 26 & 74.94 & 70.72 & 46 & 64.97 & 82.08 \\
\hline 7 & 73.15 & 82.38 & 27 & 66.54 & 85.94 & 47 & 58.07 & 73.89 \\
\hline 8 & 66.39 & 72.59 & 28 & 64.04 & 71.73 & 48 & 58.61 & 86.48 \\
\hline 9 & 78.47 & 82.84 & 29 & 62.77 & 76.18 & 49 & 65.97 & 77.02 \\
\hline 10 & 75.03 & 74.94 & 30 & 69.05 & 78.96 & 50 & 75.24 & 84.54 \\
\hline 11 & 66.35 & 80.17 & 31 & 69.21 & 75.88 & 51 & 74.20 & 66.51 \\
\hline 12 & 61.78 & 67.82 & 32 & 71.97 & 71.88 & 52 & 61.25 & 83.22 \\
\hline 13 & 78.07 & 85.00 & 33 & 66.27 & 82.85 & 53 & 71.44 & 66.65 \\
\hline 14 & 70.95 & 82.29 & 34 & 72.84 & 83.44 & 54 & 63.19 & 70.30 \\
\hline 15 & 59.47 & 74.35 & 35 & 78.79 & 75.14 & 55 & 67.16 & 72.26 \\
\hline 16 & 70.89 & 88.41 & 36 & 80.25 & 78.71 & 56 & 71.48 & 79.14 \\
\hline 17 & 81.62 & 86.18 & 37 & 74.20 & 77.30 & 57 & 65.46 & 86.44 \\
\hline 18 & 71.67 & 87.56 & 38 & 74.90 & 85.44 & 58 & 69.83 & 80.10 \\
\hline 19 & 77.46 & 80.58 & 39 & 80.40 & 78.48 & 59 & 79.46 & 68.74 \\
\hline 20 & 68.88 & 81.33 & 40 & 57.60 & 71.08 & 60 & 60.85 & 75.89 \\
\hline
\end{tabular}

who have been released from the hospital, nursing personnel are obliged to give advice on postpartum pelvic floor muscle rehabilitation exercises. The technique is as follows: the first part consists of breathing exercises. In a supine posture, the patient inhales deeply, tightens the abdomen, and then relaxes and exhales. The retraction of the anus is the second part. The patient lies supine, with both arms extended flat on both sides of the body, and the anus constricted and relaxed. 
TABLE 5: Comparison of exercise compliance.

\begin{tabular}{|c|c|c|c|c|c|c|c|c|}
\hline Number & Control group & Test group & Number & Control group & Test group & Number & Control group & Test group \\
\hline 1 & 57.24 & 48.64 & 21 & 28.74 & 78.43 & 41 & 34.24 & 70.59 \\
\hline 2 & 60.58 & 72.31 & 22 & 24.29 & 33.80 & 42 & 39.53 & 79.55 \\
\hline 4 & 74.21 & 74.80 & 24 & 50.43 & 55.63 & 44 & 47.14 & 83.04 \\
\hline 5 & 51.67 & 64.69 & 25 & 42.44 & 75.32 & 45 & 61.86 & 81.80 \\
\hline 6 & 46.70 & 57.17 & 26 & 70.12 & 75.20 & 46 & 69.31 & 68.13 \\
\hline 8 & 48.85 & 31.07 & 28 & 51.52 & 45.16 & 48 & 48.37 & 63.44 \\
\hline 9 & 71.69 & 46.17 & 29 & 47.00 & 45.24 & 49 & 38.44 & 72.44 \\
\hline 10 & 36.24 & 71.20 & 30 & 63.67 & 34.50 & 50 & 55.67 & 72.41 \\
\hline 11 & 41.44 & 74.53 & 31 & 56.18 & 44.60 & 51 & 46.38 & 55.82 \\
\hline 12 & 36.77 & 83.04 & 32 & 37.21 & 35.37 & 52 & 53.04 & 70.41 \\
\hline 16 & 62.43 & 48.75 & 36 & 33.86 & 70.07 & 56 & 41.99 & 66.35 \\
\hline 17 & 52.24 & 75.84 & 37 & 31.51 & 33.02 & 57 & 70.07 & 58.24 \\
\hline 18 & 65.03 & 71.85 & 38 & 62.98 & 45.35 & 58 & 52.11 & 64.70 \\
\hline 19 & 60.90 & 43.60 & 39 & 59.13 & 39.29 & 59 & 68.70 & 53.79 \\
\hline 20 & 58.33 & 54.66 & 40 & 60.39 & 82.84 & 60 & 52.16 & 55.12 \\
\hline
\end{tabular}

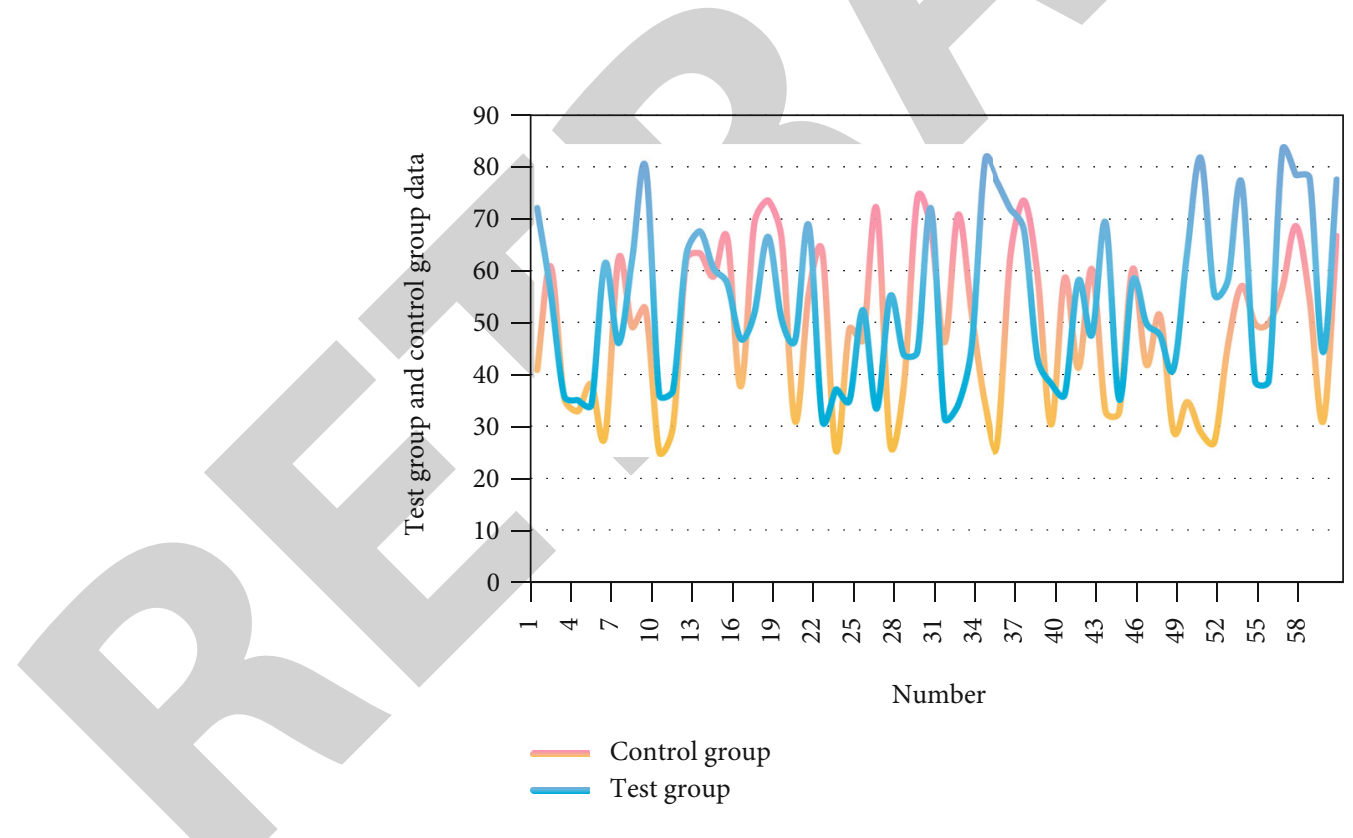

FIgURE 1: Comparison chart of comprehensive pelvic floor muscle strength.

The third section is the leg extension exercise. The patient is in a supine position, the patient's arms are stretched flat on both sides of the body, the legs are raised and raised in turn, and the angle should be 90 degrees to the body. The fourth section is abdominal and back exercises. The patient is in a supine position, the hips and legs are relaxed, the legs are slightly bent apart, and the feet are put on the bed to support the hips and back as high as possible. The fifth section is sit- ups. The patient's legs are straightened, the head and upper body are brought closer to the legs when inhaling, and the body is lying flat when exhaling. The sixth section is waist movement. The patient's knees are separated on the bed, the hands are shoulder-width apart on the bed, the elbows are at 90 degrees to the shoulders, and the waist swings from side to side. The whole-body workout is the sixth part. The patient's legs are kneeling on the bed, the arms are supported 


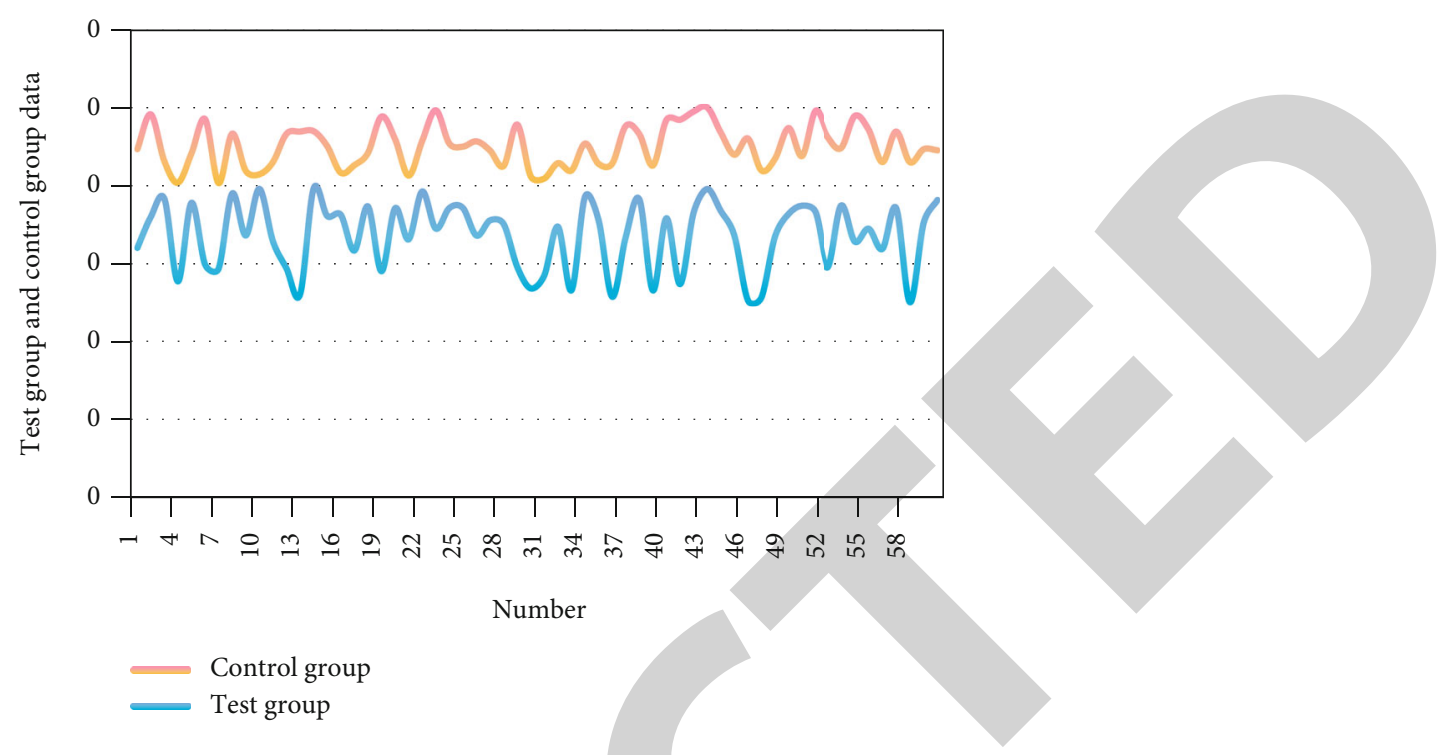

FIgURE 2: Comparison chart of the incidence of urinary incontinence.

by the hands on the bed, and the left and right legs are alternately lifted to the back. The observation group also uses the following nursing methods:

On the basis of the nursing measures of the control group, the nursing staff of our hospital carries out continuous Kegel exercise combined with Bobath ball training for the secondborn childbirth women. The specific method is as follows: (1) the members of the continuation nursing team are composed of 1 chief nurse, 1 deputy chief nurse, 2 chief nurses, and 1 nurse. They all have a bachelor's degree and have strong management, coordination, communication, expression, and technical skills. (2) The head nurse of the obstetric department serves as the team leader and is responsible for allocating tasks, including formulating continuation care plans, Kegel exercise and Bobath ball exercise continuity project planning, and pelvic floor rehabilitation effect evaluation. (3) Before the imple- mentation of the project, the research team has been trained, all have passed the assessment, can operate independently, and accurately evaluate the effect.(4) It is necessary to perform Kegel exercise combined with Bobath ball exercise. During Kegel exercise training, women need to empty their bladder first. The parturient woman can be carried out in lying, sitting, and standing positions. The anus, perineum, and urethra are contracted during inhalation, and the abdomen, buttocks, and thighs are kept relaxed without exertion. The maternal continuous contraction lasts for 3-5s and then relaxes the muscles for $5 \mathrm{~s}$ when exhaling, and each time is $20-30 \mathrm{~min}, 3$ times a day, and the breathing remains deep and slow. Bobath ball training is as follows: when the parturient takes the supine position, the parturient supports the ground with both hands and puts both feet on the ball. Both hands are on the ball, and both feet are on the ground while the parturient is in the 


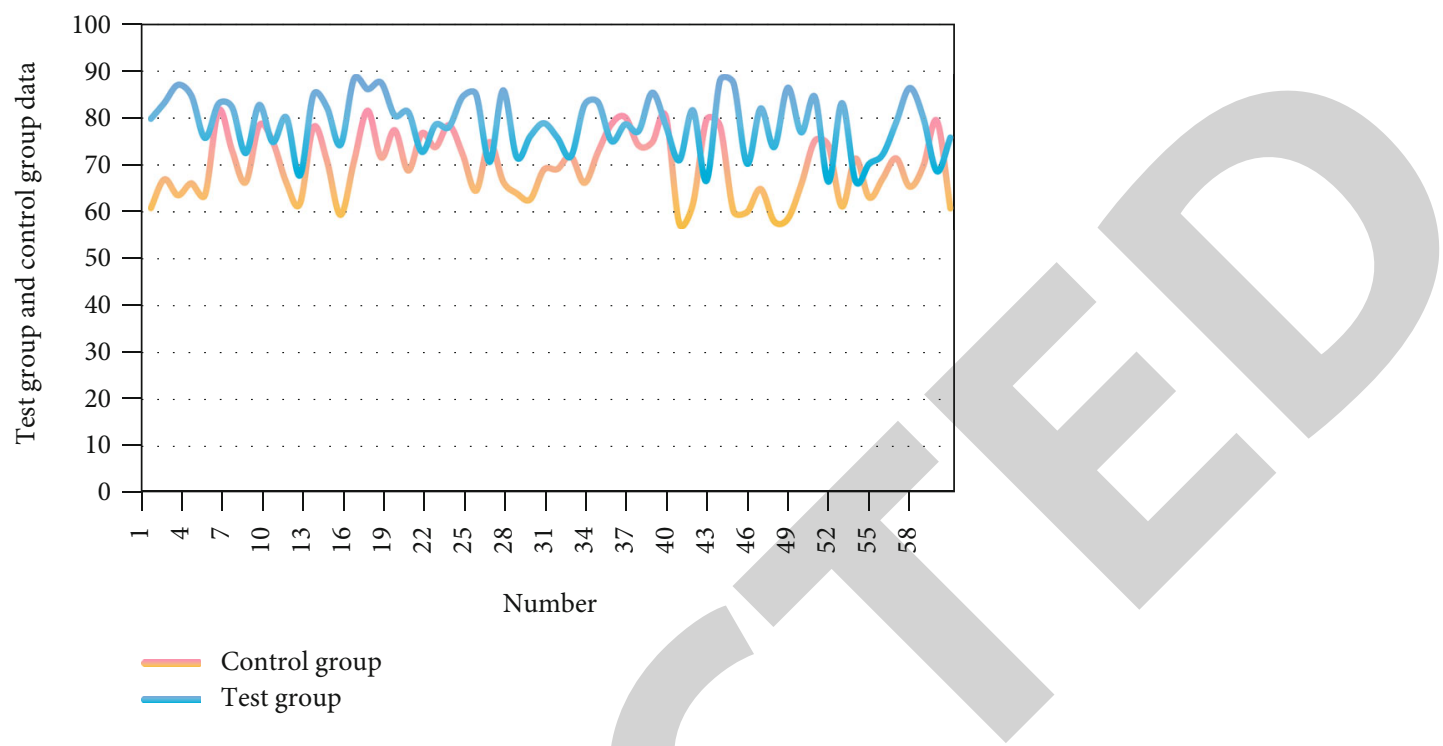

Figure 4: Comparison chart of FSFI comprehensive score.

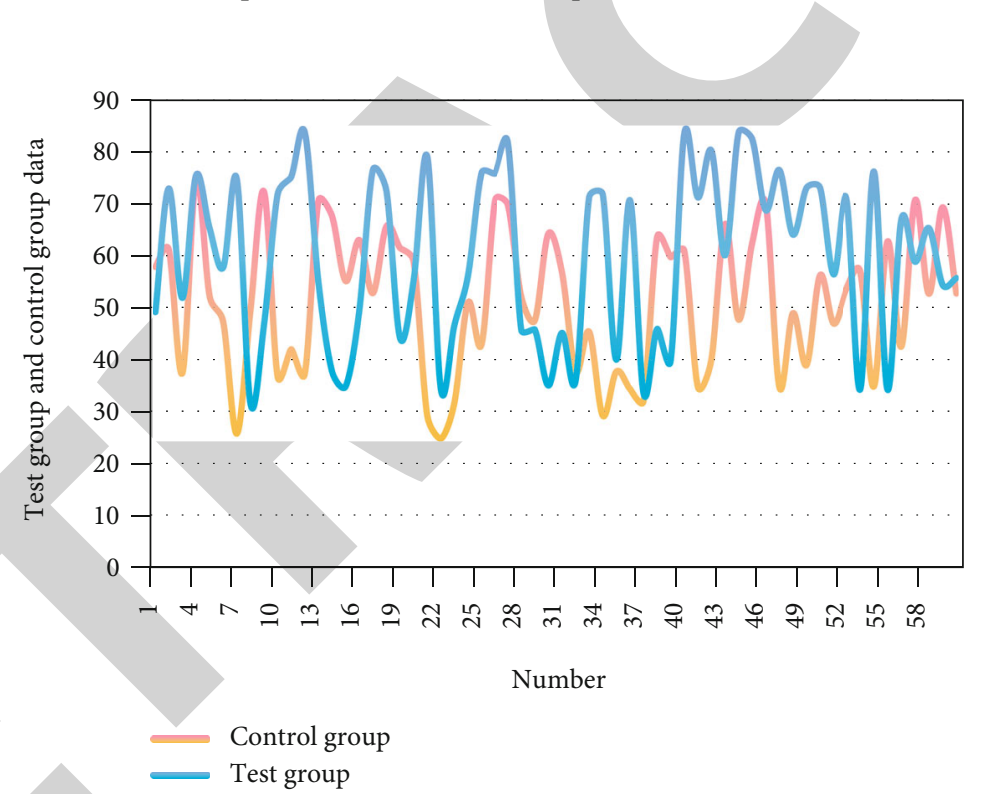

Figure 5: Comparison chart of exercise compliance.

prone posture. The parturient should maintain her back straight, tuck her abdomen, lock her legs together, and breathe steadily while doing the aforementioned two sets of motions. The parturient sits on the Bobath ball and moves up and down or shakes from side to side as she takes a seat. The range is about $10-15 \mathrm{~cm}$, and the range is suitable for the comfort of the parturient. Each position is maintained for $15-30 \mathrm{~s}, 2$ groups/day, each group rests for 5-10 s, and the frequency is 3.5 times/week. In Kegel exercise combined with Bobath ball exercise, the amount of exercise is adjusted according to personal physical ability and feeling, and the exercise lasts for 6 months.(5) Nurses need to establish personal files, such as name, parity, age, telephone number, address, pelvic floor muscle rehabilitation exercise status, and pelvic floor muscle strength rehabilitation effect.(6) Continuing care needs to be implemented by means of door-to-door guidance, telephone follow-up, WeChat group, etc. The households were followed up within 1 week after discharge and every two months. In the first month, the nurse needs to follow up by phone or WeChat once a week, and in the second month, the nurse needs to communicate and follow up by phone or WeChat every half month. In the third month, the nurse starts to follow up by phone or WeChat once a month until 6 months after delivery. The frequency of follow-up is adjusted according to the needs of the mother.

The maternal urine incontinence, pelvic organ prolapse, Kegel exercise training compliance, and pelvic floor muscle tension recovery are all assessed six months after discharge. To assess pelvic organ prolapse, the "Obstetrics and Gynecology" pelvic organ prolapse quantification (POPQ) indexing 
technique is employed. The frequency and length of maternal pelvic muscle contractions are evaluated by electromyography muscle voltage, which is classified into 1-5 levels, to determine complete pelvic floor muscular strength. The greater the intensity, the faster the pelvic floor muscle strength recovers. The International Urine Incontinence Advisory Committee's approved urinary incontinence questionnaire summary (ICI-SF) was used to assess the incidence of postpartum urinary incontinence in the two groups.

Statistical processing is as follows: in this paper, SPSS 19.0 statistical software is used for data processing. The count data is expressed as a percentage, and the $\chi^{2}$ test is performed. The measurement data is expressed as $x \pm s$, and the $t$-test is performed. The difference is statistically significant with $P<0.05$.

\section{Result}

Based on the above analysis, this paper conducts research and analysis from the perspectives of comprehensive pelvic floor muscle strength, incidence of urinary incontinence, incidence of pelvic organ prolapse, FSFI comprehensive score, and exercise compliance. The results are shown in Tables 1-5 and Figures 1-5.

\section{Conclusion}

The process of pregnancy and childbirth can cause changes in the pelvic floor support structure and damage to the pelvic floor tissues, which can easily cause pelvic floor dysfunction diseases such as stress urinary incontinence and pelvic organ prolapse. Furthermore, it is one of the most prevalent illnesses among postpartum women, and it has a significant effect on their quality of life. Strengthening pelvic floor muscle function rehabilitation training may assist the puerpera repair the nerves and pelvic floor tissue injured during pregnancy and delivery as quickly as possible, as well as enhance the pelvic floor tissue's supportive function. Therefore, it is an important measure to prevent and reduce the occurrence of pelvic floor dysfunction diseases. In addition, timely evaluation of postpartum body rehabilitation effects and resolution of existing health problems will enable women to receive the same professional training and guidance as during hospitalization after discharge. Therefore, it is necessary to carefully complete the standardized pelvic floor muscle exercises to improve the comprehensive muscle strength of the pelvic floor. To sum up, we need to carry out pelvic floor muscle function recovery exercise training guidance to promote the recovery of postpartum pelvic floor muscle strength, improve the compliance of the pelvic floor muscle exercise of the parturient, and reduce the occurrence of urinary incontinence and pelvic floor organ prolapse.

\section{Data Availability}

The data used to support the findings of this study are included within the article.

\section{Conflicts of Interest}

The authors declare that there is no conflict of interest regarding the publication of this paper.

\section{Acknowledgments}

This work was supported by the Project of Medical and Health Science and Technology of Zhejiang Province (study on the effect of family group pregnancy health care mode incorporating birth dance on the pelvic floor function rehabilitation of primiparas after delivery. No. 2021KY880), the Project of Medical Health Science and Technology Project of Zhejiang Province (clinical applied research of early neonatal basic health care in family group pregnancy care model. No. 2019KY128), and Medical Key Discipline of Hangzhou.

\section{References}

[1] M. Mert Karadas and F. Terzioglu, "The impact of the using high-fidelity simulation and standardized patients to management of postpartum hemorrhage in undergraduate nursing students: a randomized controlled study in Turkey," Health Care for Women International, vol. 40, no. 5, pp. 597-612, 2019.

[2] C. Linar, M. Badiran, and R. Hanum, "The study of nursing during the postpartum period from the perspective of Aceh Culture in Gampong Asan Kareung, Lhokseumawe City," Journal La Medihealtico, vol. 1, no. 5, pp. 30-39, 2020.

[3] F. Ay, E. Tektaş, A. Mak, and N. Aktay, "Postpartum depression and the factors affecting it: 2000-2017 study results," Journal of Psychiatric Nursing, vol. 9, no. 3, pp. 147-152, 2018.

[4] J. M. Stern, M. Konner, T. N. Herman, and S. Reichlin, "Nursing behaviour, prolactin and postpartum amenorrhoea during prolonged lactation in American and Kung mothers," Clinical Endocrinology, vol. 25, no. 3, pp. 247-258, 1986.

[5] Y. C. Yeh, W. St John, and L. Venturato, "Inside a postpartum nursing center: tradition and change," Asian Nursing Research, vol. 10, no. 2, pp. 94-99, 2016.

[6] I. D. S. S. Strefling, C. B. Borba, C. C. Demori, M. C. Soares, C. H. G. J. Váz, and C. P. D. Santos, "Perceptions of puerperas on nursing care in joint accommodation," Revista de Pesquisa: Cuidado é Fundamental Online, vol. 9, no. 2, pp. 333-339, 2017.

[7] C. A. Wollenhaup, E. L. Stevenson, J. Thompson, H. A. Gordon, and G. Nunn, "Implementation of a modified bedside handoff for a postpartum unit," JONA: The Journal of Nursing Administration, vol. 47, no. 6, pp. 320-326, 2017.

[8] G. P. Mensah, W. ten Ham-Baloyi, D. van Rooyen, and S. Jardien-Baboo, "Guidelines for the nursing management of gestational diabetes mellitus: an integrative literature review," Nursing Open, vol. 7, no. 1, pp. 78-90, 2020.

[9] H. M. Alharqi and J. A. Albattawi, "Assessment of knowledge and attitude of women towards postpartum exercise," IOSR Journal of Nursing and Health Science, vol. 7, no. 1, pp. 1620, 2018.

[10] P. P. Mano, R. Samson, Jayasankari et al., "A study to explore the factors contributing to the development of postpartum blues among postnatal mothers at Pondicherry Institute of Medical Sciences, Puducherry," International Journal of Advances in Nursing Management, vol. 6, no. 4, pp. 309-312, 2018. 
[11] R. A. Elshatarat, M. I. Yacoub, Z. T. Saleh et al., "Perinatal nurses' and midwives' knowledge about assessment and management of postpartum depression," Journal of Psychosocial Nursing and Mental Health Services, vol. 56, no. 12, pp. 3646, 2018.

[12] Y. C. Yeh, W. St John, Y. H. Chuang, and Y. P. Huang, "The care needs of postpartum women taking their first time of doing the month: a qualitative study," Contemporary Nurse, vol. 53, no. 5, pp. 576-588, 2017.

[13] M. M. McKelvey and J. Espelin, "Postpartum depression," Nursing Made Incredibly Easy, vol. 16, no. 3, pp. 28-35, 2018.

[14] Y. H. Wu, Y. J. Ho, J. P. Han, and S. Y. Chen, "The influence of breastfeeding self-efficacy and breastfeeding intention on breastfeeding behavior in postpartum women," $\mathrm{Hu}$ li $\mathrm{za}$ zhi The Journal of Nursing, vol. 65, no. 1, pp. 42-50, 2018.

[15] F. F. Chung, G. H. Wan, S. C. Kuo, K. C. Lin, and H. E. Liu, "Mother-infant interaction quality and sense of parenting competence at six months postpartum for first-time mothers in Taiwan: a multiple time series design," BMC Pregnancy and Childbirth, vol. 18, no. 1, pp. 1-13, 2018.

[16] M. Horibe, Y. Hane, J. Abe et al., "Contraceptives as possible risk factors for postpartum depression: a retrospective study of the food and drug administration adverse event reporting system, 2004-2015," Nursing Open, vol. 5, no. 2, pp. 131138, 2018.

[17] S. H. Kim and K. M. Sung, "The effects of auricular acupressure on depression and fatigue among early postpartum mothers," Journal of East-West Nursing Research, vol. 25, no. 2, pp. 91-102, 2019.

[18] C. T. Beck, "Mother-infant interaction during postpartum depression: a metaphor analysis," Canadian Journal of Nursing Research, vol. 52, no. 2, pp. 108-116, 2020.

[19] M. Hakimi, M. Kheirkhah, J. Abolghasemi, and R. Hakimi, "The effects of competency-based education on midwifery students knowledge, skills, and self-confidence for postpartum hemorrhage management," Nursing and Midwifery Studies, vol. 10, no. 3, pp. 137-144, 2021.

[20] L. S. Goshin, D. G. Sissoko, G. Neumann, C. Sufrin, and L. Byrnes, "Perinatal nurses' experiences with and knowledge of the care of incarcerated women during pregnancy and the postpartum period," Journal of Obstetric, Gynecologic \& Neonatal Nursing, vol. 48, no. 1, pp. 27-36, 2019.

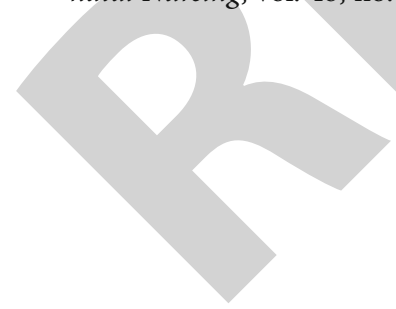

